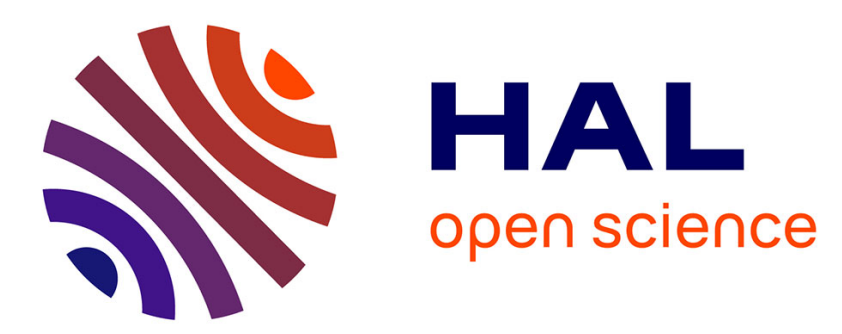

\title{
Dependability Evaluation of a Replication Service for Mobile Applications in Dynamic Ad-Hoc Networks
}

Erling V. Matthiesen, Ossama Hamouda, Mohamed Kaâniche, Hans-Peter

Schwefel

\section{- To cite this version:}

Erling V. Matthiesen, Ossama Hamouda, Mohamed Kaâniche, Hans-Peter Schwefel. Dependability Evaluation of a Replication Service for Mobile Applications in Dynamic Ad-Hoc Networks. 5th International Service Availability Symposium, ISAS 2008, May 2008, Tokyo, Japan. pp.171-186, 10.1007/978-3-540-68129-8_14. hal-00851873

\section{HAL Id: hal-00851873 \\ https://hal.science/hal-00851873}

Submitted on 23 Aug 2013

HAL is a multi-disciplinary open access archive for the deposit and dissemination of scientific research documents, whether they are published or not. The documents may come from teaching and research institutions in France or abroad, or from public or private research centers.
L'archive ouverte pluridisciplinaire HAL, est destinée au dépôt et à la diffusion de documents scientifiques de niveau recherche, publiés ou non, émanant des établissements d'enseignement et de recherche français ou étrangers, des laboratoires publics ou privés. 


\title{
Dependability Evaluation of a Replication Service for Mobile Applications in Dynamic Ad-Hoc Networks*
}

\author{
Erling V. Matthiesen ${ }^{1}$, Ossama Hamouda ${ }^{2,3}$, \\ Mohamed Kaâniche ${ }^{2,3}$, and Hans-Peter Schwefel ${ }^{1,4}$ \\ ${ }^{1}$ CTIF, Aalborg University, Niels Jernes Vej 12/A5-212 \\ 9220 Aalborg-Øst, Denmark \\ \{evm, hps $\}$ @es . aau.dk \\ ${ }^{2}$ CNRS; LAAS ; 7, avenue du Colonel Roche, F-31077 Toulouse, France \\ ${ }^{3}$ Université de Toulouse ; UPS, INSA, INP, ISAE ; LAAS-CNRS : Toulouse, France \\ \{ossama. hamouda, mohamed. kaaniche\} @laas.fr \\ ${ }^{4}$ Forschungszentrum Telekommunikation Wien - FTW, Donau - City Straße 1, \\ 1220 Vienna, Austria
}

\begin{abstract}
In order to increase availability and reliability of stateful applications, redundancy as provided by replication in cluster solutions is a wellknown and frequently utilized approach. For mobile services in dynamic ad-hoc networks, such replication mechanisms have to be adapted to deal with the frequently higher communication delays and with the intermittent connectivity. Dynamic clustering strategies in which the replica set is adjusted to the current network state can help to handle the network dynamicity. The paper develops a stochastic Petri net model (and its corresponding Markov chain representation) to analyze the resulting availability and replica consistency in such dynamic clusters. The numerical results are interpreted in the context of a vehicular (c2c) communication use-case and can be used to determine optimized cluster configuration parameters.
\end{abstract}

\section{Introduction}

Many of the future networking scenarios consist both of wireless multi-hop parts and infrastructure based network components. For new application types and future service platforms, server-based applications access is not only offered by the infrastructure network part, but also by the potentially mobile nodes in the ad-hoc domain. An example for such service provisioning scenarios is the vehicular communication setting [2], in which cars will be able to communicate with each other. This communication will be used for safety critical applications that require high availability. For applications used in automotive traffic this is especially true since application failure could affect driver behavior or directly affect the state of the car, e.g., spurious application of brakes. Traditional solutions for high-availability rely on redundancy offered by cluster implementations, in which Middleware services [1] support the timely replication and fail-over in case of crash failures of individual

* This work was partially supported by the HIDENETs project (EU-IST-FP6-26979).

T. Nanya et al. (Eds.): ISAS 2008, LNCS 5017, pp. 171-186, 2008.

(C) Springer-Verlag Berlin Heidelberg 2008 
cluster nodes [7]. For stateful applications, such fail-over capability typically involves timely replication of application state, which could be implemented by a redundant distributed shared memory [5].

In mobile ad hoc networks (MANETs) the lifetime of a communication path may be short [2]. Communication delays in principle are unbounded due to the unpredictability of the Medium Access procedures on the link-layer and potential rerouting delays in dynamic multi-hop scenarios [9]. Replication strategies for dynamic data such as application state need to take these communication properties into account [8]: Larger communication delays can increase the probability of inconsistent replica state, so that dynamic cluster member selection can lead to substantial improvements in mobile scenarios [12]. The replication manager (RM) defined in [3] an example of such a middleware component that provides applications with a resilient shared memory area and performs management of such a dynamic cluster. Heuristic algorithms as investigated in [8], based on measured communication delays and geographic positioning and speed information, can be utilized to trigger membership reconfiguration in a mobile ad-hoc network setting.

In this paper, we evaluate the dependability of a replicated stateful application service based on dynamic cluster formation as provided by the Replication Manager. The metrics we evaluate are the number of participating nodes in the cluster, replica data consistency, and application availability. The former can be an important metric to improve client-access to the replicated service in delay-constrained scenarios, as client-nodes can potentially select the server instance with shortest communication delay to the clients [10]. Replica consistency is expressed as the number of nodes that store the correct data, where correctness here refers to the real-time ordering of write operations to the distributed memory area storing the changing application state.

Using numerical results from analytic Stochastic Petri Net models of this dynamic replication scenario, we experiment with different geographic mobility types, and different degrees of dynamicity of the application state [6].

The rest of the paper is organized as follows. Section 2 describes basic background about: a) typical applications and use scenarios that illustrate the context of the study, b) replication management and data consistency related problems and challenges, and c) the main concepts behind the design of the replication manager investigated in this paper. The dependability models of the replication manager allowing the assessment of the probabilities characterizing the number of replica in the network and their consistency are presented in Section 3. Numerical and sensitivity analysis results are discussed in Section 4. Finally, Section 5 summarizes the main conclusions and discusses future work.

\section{Background}

In this section we present necessary background information needed to understand the scenarios investigated in this paper, both with respect to replication and vehicular adhoc networks. First we describe the scenario and then we go into the problem of data consistency in a distributed shared memory area and also the problems involved with service replication in ad-hoc networks. 


\subsection{Scenario Description}

Various applications in vehicular settings could benefit from the replication service investigated in this paper [11]. We can consider as an example, an ad-hoc network based road-traffic information service, whose information base is dynamically updated. Utilizing the Replication Manager middleware service, the application state (traffic information) is kept in a memory area which is shared among the participating servers in the replica set. In case the network topology (connectivity graph) changes significantly, e.g., when a server node is exiting the highway, the intra-cluster communication with that node will experience increasing delays as the geographic distance and eventually the multi-hop communication path length grows. As the communication with a node deteriorates, the probability of an inconsistent application state will increase.

The model presented in this paper and the sensitivity analysis results are intended to quantify and investigate the impact of different traffic situations and dynamic behaviors on the resulting application availability and the probability of state inconsistency of the participating servers in replica set.

In a replication manager use case, three different nodes and roles can be distinguished: user nodes, relay nodes and service nodes. The user nodes are the clients of the application service which is provided by the service nodes. Although different replica sets can co-exist and overlap, the subsequent discussions will without loss of generality focus on a single replicated service instance. In order to start using the replicated application service, the RM middleware will need to identify the corresponding replica node set, e.g., via a dynamic naming service. Upon crash failure of the service node or degraded/disconnected communication properties, the RM service in the user node can failover to other service nodes in the replica group. Relay nodes are network nodes that relay packets in the network in a multi-hop communication scenario. In principle, all service nodes within the reach of the multihop connectivity are eligible to act as application service replicas; however, in order to limit communication delays between replica nodes, the communication pathlengths may be bounded to a maximum hop-count $\mathrm{H}$; in the extreme case $\mathrm{H}=1$, only direct neighbors (within link-layer connectivity) would be eligible to act as replicas. Any replica node can modify the data in the shared memory area (e.g., in reaction to processing requests by users).

In the rest of the paper we will refer to the maximum number of eligible replica nodes in the network as $n$, and to the number of service nodes active in the replica group as $k$.

\subsection{Background on Data Consistency}

Dynamic application state (as in the traffic information example) requires timely update and replication of the stored data. The replication model used in the RM is a simple shared memory area. As a node is taken into the replica group, it becomes accessible as a server to the users. Consistent data replication implies that all updates must have been received from all other nodes in the group before a node can be considered consistent. A typical shared memory area involves locking mechanisms to ensure that the memory is consistent at the time of a write operation. Locking or other 
concurrency or consistency control mechanisms however require communication overhead and cause additional delays. Furthermore, in scenarios of unbounded communication delays and for short-lived connectivity relations, the design of such mechanisms is very challenging. Due to the unreliability of the communication channel in mobile ad-hoc networks we choose to design the RM with optimistic replication and no concurrency control mechanisms. The sensitivity analysis provided in this paper shows the feasibility of this approach and gives guidelines as to which scenarios the RM will be able to provide a dependable dynamic shared memory area to the application. Omitting concurrency control is a choice to reduce replication overhead. The models presented in this paper show that replication is possible without concurrency control under certain circumstances.

\subsection{Replication Management Problems and Proposed Design Concepts}

In a car-to-car ad-hoc network the topology may change so frequently that the amount of signaling needed to keep a group of replicas consistent is potentially large [13]. The smaller the number of active replicas, the higher the unavailability of the application service due to crash failures of the server (which is undistinguishable from the server leaving the connected network). On the other hand service replication by broadcasting state information to all nodes in the network will increase wireless bandwidth consumption and may lead to congestion. The number of replicas to select is hence a tradeoff between low overhead and availability. The design goal of the RM is to provide services to the user with a high perceived quality of service, while keeping replication and reconfiguration overhead as low as possible. Therefore we have chosen to favor the tradeoffs that give the best perceived service to the user node.

The metrics with the highest impact on user perceived quality of service are service response time, service availability, and the correctness of the service [4]. Correctness is influenced by the consistency of the replicated data. If a user is getting a service which is provided based on out-of-date data the service is not correct. Incorrect service influences the user's perception of the service dependability. The faults that affect the user perceived quality of service are closely related to the metrics already described. For example packet loss or excess delay affects the timeliness of the service and also the correctness. A lost update packet is extending the time where the replica server is in an inconsistent state until a successful retransmission or a new update message is received. The same is true for large delay. If a single server in the group is experiencing these types of faults, it can be excluded from the group and a new service node - if any eligible ones reachable in the network - can be included. The replicas are selected in order to achieve stable clusters as this is preferred to minimize reconfiguration overhead; see [1] for strategies to increase stability.

In summary the most important problems addressed by the RM are:

- $\quad$ Selection of replicas with stability criteria and best communication metrics.

- Reduction of reconfiguration overhead.

- Reduction of service response time.

Any node in the replica group can propose new group members; when a member is proposed all existing members must acknowledge the adoption of the new members. 
If the majority of the existing members accept the new group member by sending a positive acknowledgement to the proposing node, the latter will send an updated membership list to all nodes in the group and to all user nodes using the service provided by the group. The user nodes need to get member updates in order to have an up to date list of failover candidates in case they loose their connection with the server they are already using. The state manager part of the replication manager is responsible for sending update messages in case of a state change caused by a write operation to the shared memory region, to store received state variables and to send an acknowledgement when a state update message has been received. In this way the write operation will be replicated to the other group members. The retrieval part is responsible for selecting a server to be used by the user nodes in case they loose the connection with the server they were already using. The retrieval part of the RM is storing the updated list of group members and selects the failover server based on communication metrics and geographic properties of the servers. Furthermore the retrieval part of the RM is responsible for retrieving the state of the shared memory area when a new service node joins the replica group.

\section{Dependability Modeling for Replica Consistency}

In this section, we present the methodology and the approach of our dependability modeling. Moreover, the objective discussed in this section is to develop a method and a model making it possible to evaluate quantitative measures characterizing the consistency of the dynamic application state in the replica group. Markov chains and generalized stochastic Petri nets (GSPNs) [15] are commonly used to perform dependability evaluation studies and sensitivity analyses aimed at identifying parameters having the most significant impact on the measures. When using analytical techniques e.g., GSPNs and Markov chains, the system must be described at a high level of abstraction. Simplified assumptions are generally needed to obtain tractable models. Although simulation can be used to describe the system at a more detailed level, it is more costly in terms of the processing time needed to obtain accurate and statistically significant quantitative results.

This section develops GSPN models considering exponential assumptions for the underlying stochastic processes. Although such assumptions may not faithfully reflect reality, the results obtained from the models and sensitivity analysis give preliminary indications and estimations about the expected behaviors and trends that can be observed. More accurate results can be obtained considering more general distributions, using for example Matrix-Exponential distributions [14] or non Markovian models.

In the following, we represent a generic GSPN model and the corresponding Markov chains [5]. Then, we present the quantitative measures evaluated from the models to assess the data consistency and application availability. Finally, we discuss the main parameters that are considered in the sensitivity analysis.

\subsection{GSPN Model for the Replica Consistency Estimation}

Fig.1 represents the proposed GSPN model describing the potential evolution of replication group size and inconsistency. The model has four input parameters. The 


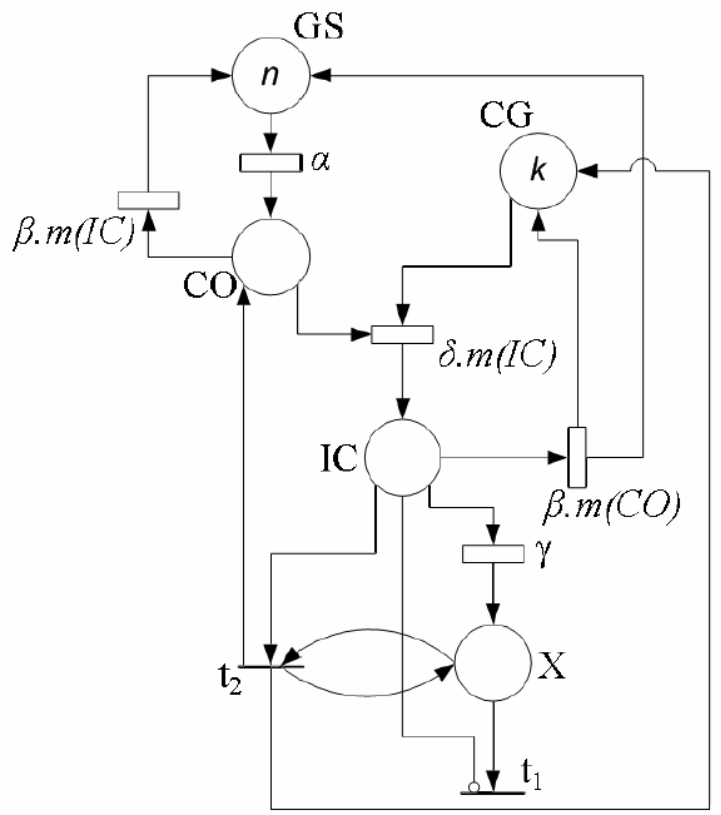

Fig. 1. GSPN model of network size $n$ and replication group size $k$

first parameter denoted by $\alpha$ is the rate at which new eligible service nodes enter the (multi-hop) communication range of the existing group. This parameter depends on the geographic mobility model, the link-layer characteristics of the wireless technology (e.g., expressed by a communication radius), and the criterion on 'eligibility' to act as cluster node, e.g., the max hop-count $\mathrm{H}$ of the multi-hop connection. The second parameter, $\beta$, is the rate with which eligible service nodes leave the connectivity range of the replica set; $\beta$ is influenced by the same three factors as stated above for $\alpha$. The third parameter, $\gamma$, is the rate with which the state of the application service changes. This change rate obviously depends on the application type and the service usage scenario, hence may be influenced by the number of client nodes. The fourth parameter, $\delta$, is the rate that represents the network and processing delay of an update message between replica nodes.

There are four main places in the Petri net; the two most important ones are labeled $I C$ and $C O$. IC and $C O$ denote the number of inconsistent and consistent nodes in the group, respectively. The top place $G S$ is initially marked with a number of tokens corresponding to the network size $n$, i.e., the maximum number of nodes of the network. The initial marking of the place $C G$ specifies the desired number of replica in the group $(k)$. The place $X$ is a utility place that moves all tokens from $C O$ to $I C$ and $C G$ when the $\gamma$ transition fires i.e., when the original data are updated. When place $X$ gets marked, we remove all the tokens found in place $C O$ by activating $t_{2}$. When $C O$ is empty, we remove all the tokens placed in place $X$ by activating $\mathrm{t}_{1}$. Transitions $t_{1}$ and $t_{2}$ are instantaneous whereas all the other transitions are exponentially distributed timed transitions. It is important to note that the firing rates 


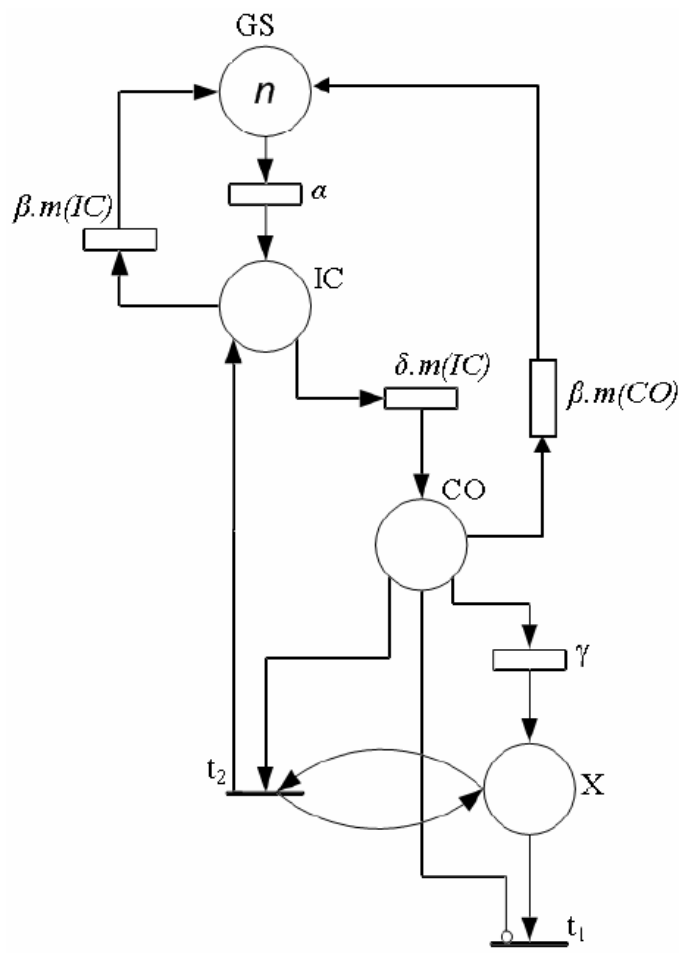

Fig. 2. GSPN model of replication group with $n=k$

of transitions $(\delta)$ and $(\beta)$ are weighted by the marking $m(I C)$ and $m(C O)$ of the input places $I C$ and $C O$, respectively.

The GSPN model of Fig. 1 can be simplified when $n=k$. This is illustrated in Fig. 2.

\subsection{Markov Model Derived from GSPN}

The Markov models describing the evolution of replica group members and their consistency are derived automatically from the corresponding GSPN models. For example, the Markov model derived from the GSPN model depicted in Fig.3 with $n=k$ is given in Fig.4.

The Markov chain depicted in Fig.3 reflects an increasing number of replica group members when moving to the right within the columns. The number of consistent group members is depicted in the rows, increasing from top to bottom. Note that for illustration purposes, a 3-dimensional state-space labeling is used; the third component, the number of 'free' places in the replica set, is actually fully determined by the first two components.

The state in the lower right hand side is the optimal state of the replica group, as the maximum number $n$ of consistent replica nodes is present. Fully consistent replica groups (of not necessarily maximal size) are represented by the whole lower diagonal. 


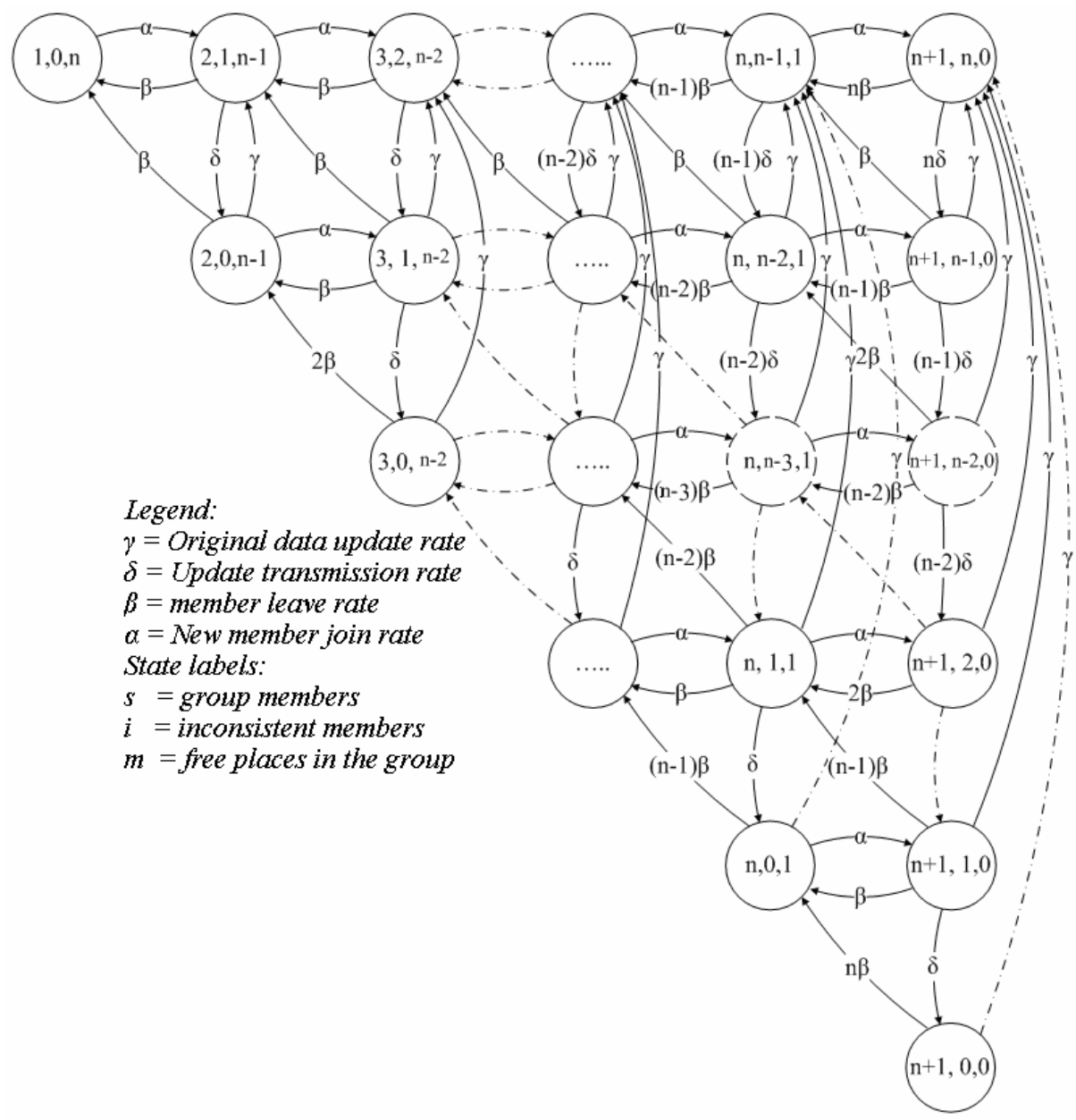

Fig. 3. Markov model of the GSPN model

The GSPN model of Fig. 2 is generic and can be used to automatically generate the Markov chain associated with any $(n)$ group size. As an example, Fig.4 shows the Markov chain representing replication consistency with $n=k=2$ as group size. The arrow labels represent the rate of the corresponding transition; for instance, an arrow labeled " $\beta$ " represents the leaving rate of a node from a group, regardless of whether this node is leaving because of a crash failure or another cause.

In the Markov model of Fig. 3, each state is labeled with three parameters $(s, i, m)$ where $s$ denotes the group size, $i$ denotes the number of inconsistent members and $m$ denotes the number of free places in the group, i.e., the number of members that can still join the group. We distinguish three main sets of states:

- The states labeled " $i=0$ " denote those where all members of group at certain time are consistent. 


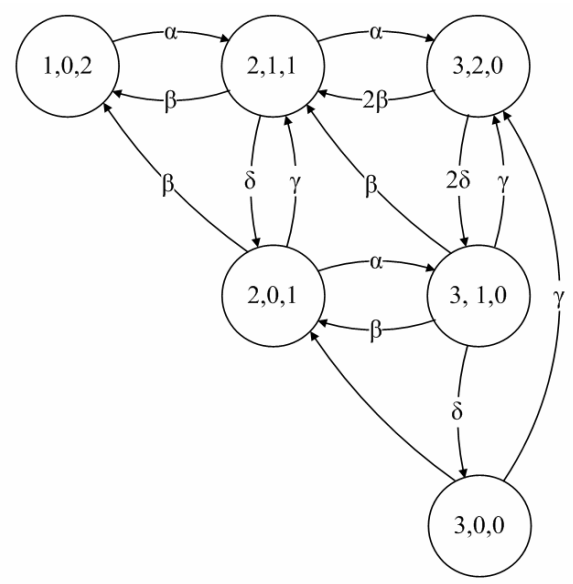

Fig. 4. Markov model of the GSPN model for $n=2$

- The states labeled " $s=n+1, m=0$ " represented in the last column denote those where the system has no more free places and there are " $s-i$ " members which are consistent.

- The state labeled " $n+1,0,0$ " denote that the system is saturated with the maximum number of members and all are consistent.

From this model some interesting formulas can be derived; namely the probability of all existing group members being consistent. In the figure, this is the probability of the Markov chain being in any of the states in the lower diagonal of states. Using the regular structure of the Markov chain, this probability can be found by the expression:

$$
P(\text { cons })=\frac{1}{\sum_{j=0}^{n}\left(\frac{\alpha^{j} \beta^{(n-j)}}{j !}\right)} \sum_{i=1}^{n} \frac{(\alpha \delta)^{(n-i+1)} \beta^{(i-1)}}{\prod_{k=1}^{n-i+1}(k(\beta+\delta)+\gamma)}
$$

The behavior of the size of the replica set (expressed by the first component, $s$, of the state-space) is actually fully determined by the rates of arrivals and departures from the group ( $\alpha$ and $\beta$ respectively). As this is a birth-death process, the probability of a certain replica size is equivalent to the queue-length probability of an $M / M / n / n$ queuing system, which can be found in any standard queuing theory book to be [16]:

$$
P(n, \beta, \alpha)=\frac{\alpha^{n}}{n ! \beta^{n} \sum_{i=0}^{n} \frac{1}{i !}\left(\frac{\alpha}{\beta}\right)^{i}}=\frac{1}{\sum_{i=0}^{n} i ! C_{i}^{n}\left(\frac{\beta}{\alpha}\right)^{i}}
$$

Hereby, $C_{i}^{n}=\frac{n !}{i !(n-i) !}$ denotes the binomial coefficients. Note that although Equation (2) can be derived directly from a simple $\mathrm{M} / \mathrm{M} / \mathrm{n} / \mathrm{n}$ model, this is not the case any 
more for the more complex 2-dimensional Markov chain structure that lead to the derivation of Eq. (1).

In Eq. (3) the service availability is calculated given that a specific user perceives a specific server with the availability $A_{s}$.

$$
A=\sum_{i=1}^{k} P(i, \beta, \alpha) \cdot\left(1-\left(1-A_{S}\right)^{i}\right)
$$

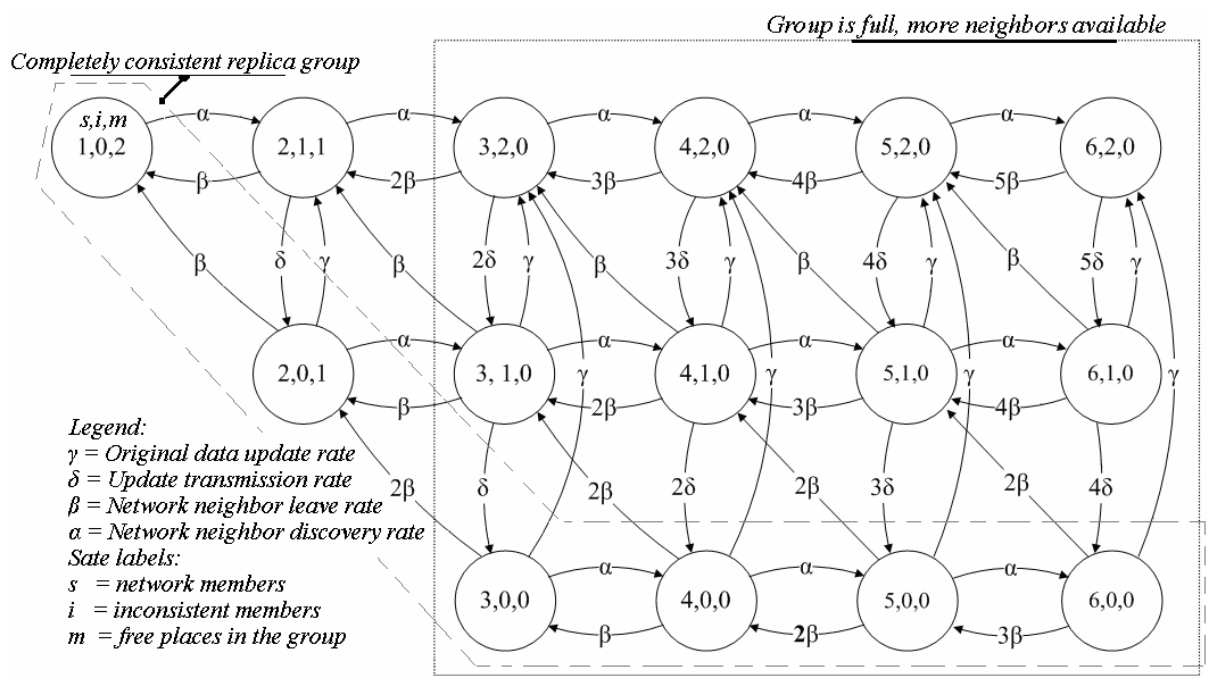

Fig. 5. Markov model with network size $n=5$ and replica group $k=2$

Fig. 5 shows another example with $n=5$ and $k=2$. The number of reachable neighbors is given by $(n-k)$. In the first part of the figure, the group is being established by nodes entering the network. The part marked as the group being full is added to make the model reflect better the dynamics of a fixed maximum size group, being maintained in a variable size connected vehicular ad-hoc network. In this part of the model nodes from the known neighborhood of the replica group are used as replacements when a node leaves the group.

\section{Results}

This section presents some numerical results for sensitivity analysis for the probabilities derived in Eq. (1), Eq. (2) and Eq. (3). We first discuss the values assigned to the parameters of the models and then present some results illustrating the behavior of the probabilities characterizing the inconsistency among group replica, the probability of reaching the maximum group size, and the service availability. 


\subsection{Parameter Values}

Naturally there are constraints on the rates used in the model if the resulting numbers should show results of realistic scenarios. For instance the update rate is used to represent the end-to-end delay of the network between the nodes in the group. There is a theoretical minimum to this delay which is the time it takes to transfer one update message to a link-layer neighbor. Assuming that one update message can be sent within 50ms under good conditions (one hop, no MAC delay) the corresponding update rate will be around $\delta=1200 /$ minute. To analyze the impact of this parameter on the results, two different values of $\delta$ are considered: 1000 and 100 per minute. The order of magnitude of the data change rate $\gamma$ depends on the considered application. Two different values of this parameter have been considered for the results presented in this section: 10 and 100 per minute, corresponding to an average time between two consecutive updates of $6 \mathrm{sec}$. and $600 \mathrm{msec}$. respectively.

As regards the rate $\alpha$ of meeting new cars that are able to join the group, we have also considered different values corresponding to different traffic situations. The minimum rate is zero meaning not meeting any cars at all on the road. An example of value of the rate $\alpha$ is $30 / \mathrm{sec}$ which is approximately equivalent to cars, with the length of 5 meters, driving in opposite directions with a speed of $200 \mathrm{~km} / \mathrm{h}$ with little or no space between the cars. This value could be much higher when considering multi-hop communication. Various values for the leaving rate $\beta$ can also be considered to reflect different behaviors of the participating nodes. This value is also difficult to estimate because it depends on several influencing factors.

Rather than focusing on the absolute values of $\alpha$ and $\beta$, one can analyze the results by considering the relative ratio $\alpha / \beta$. Higher values of this ratio correspond to environments where the probability of meeting a new car is higher than the probability that a participating node will leave the network. In a freeway scenario where cars join and leave the road via on and off-ramps and cars travel with a mean speed of $130 \mathrm{~km} / \mathrm{h}$ excluding trucks the rate of cars joining the group is quite low because the cars travel with approximately the same speed. Assuming three cars joining the group per minute gives a join rate of $3 /$ minute. With an assumption that each car stays in the group on average for 3 minutes the actual leave rate is $\beta=0.33 /$ minute. Accordingly the ratio $\alpha / \beta$ will be around 10 . Much higher values can be obtained for other scenarios.

To summarize the settings of the parameters $\alpha, \beta, \delta$, and $\gamma$ that represent the range of values expected to be found using measurements from vehicular networks would be as presented in Table 1 .

Table 1. The maximum and minimum values for each parameter

\begin{tabular}{|c|c|c|c|c|}
\hline Parameter & $\alpha$ & $\beta$ & $\gamma$ & $\delta$ \\
\hline Min & 0 & 0 & 10 & 100 \\
\hline Max & 10000 & 10000 & 100 & 1000 \\
\hline
\end{tabular}




\subsection{Sensitivity Analyses for Replica Consistency}

Fig. 6 plots some numerical results obtained by Eq. (2). As expected, it is shown that it is harder to reach the maximum group size for larger groups. If more members are needed to form a full group it is more difficult to do that given a constant arrival of new possible group members. The probability plotted in Fig.6 is only affected by the values of $\alpha$ and $\beta$ that characterize the dynamics of the network; the higher the value of the ratio $\alpha / \beta$, the closer the probability of reaching the maximum group size gets to the value 1 . Also, it can be seen that this probability is very sensitive to the value of $\alpha / \beta$ when this ratio is relatively low (less than $10^{2}$ in the example setting).

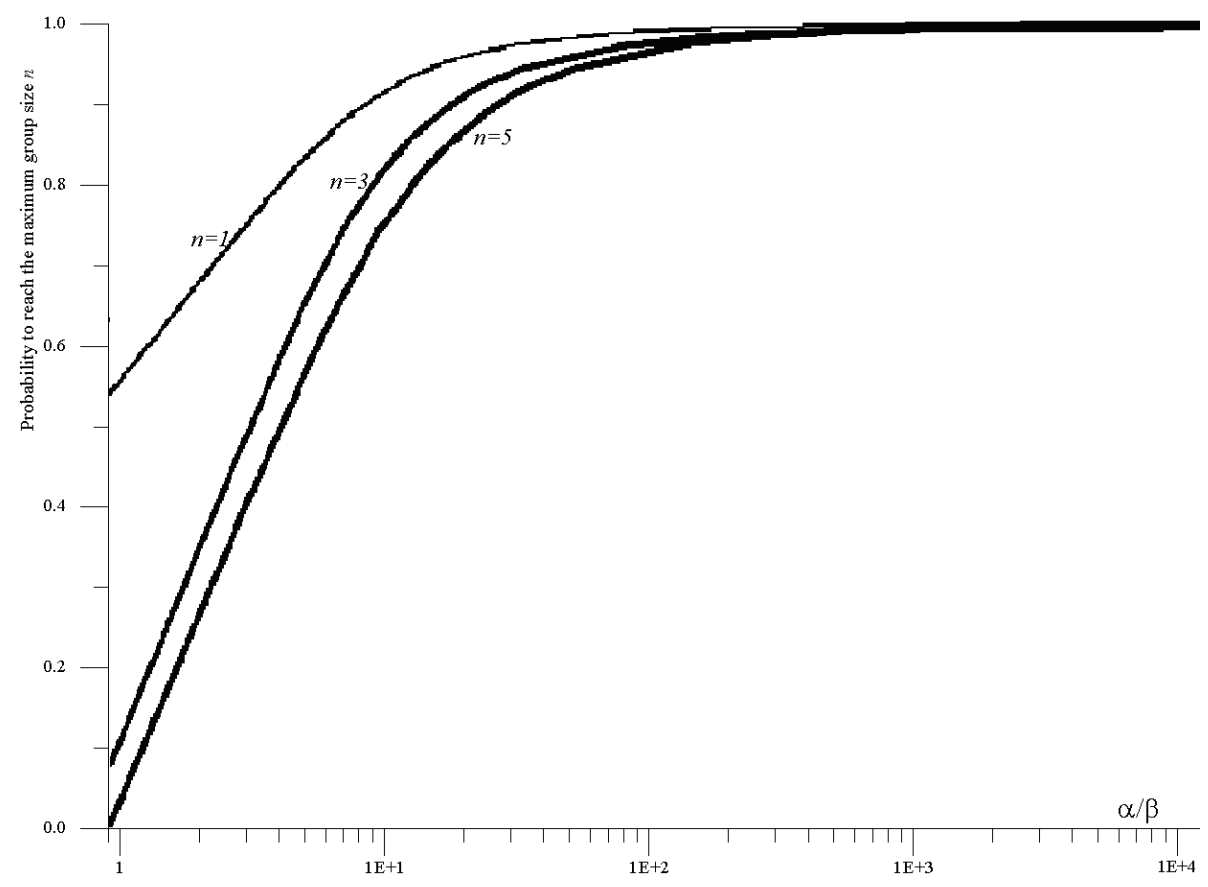

Fig. 6. Probability of presence of maximum group size $n=k$

As regards the replicas consistency, Figure 7 shows that the probability of a consistent replica set converges rather fast to a limit value for increasing $\alpha / \beta$. This limit value depends strongly on the relation of the application state change, $\gamma$, and the network delay rate, $\delta$. This is intuitive too, since timely updates are needed to achieve consistent replica groups.

Figure 7 shows the combined impact of parameters $\gamma$ and $\delta$ on the consistency probabilities. It can be seen that a decrease of $\delta$ of one order of magnitude (from 1000 to 100), could lead to a degradation of the consistency probability in the order of 4 to 9 times, depending on the considered values for the ratio $\alpha / \beta$ and the value of $\gamma$. Also, for $\alpha / \beta$ values higher than 10 , the higher the ratio $\delta / \gamma$.the higher the consistency probability. 


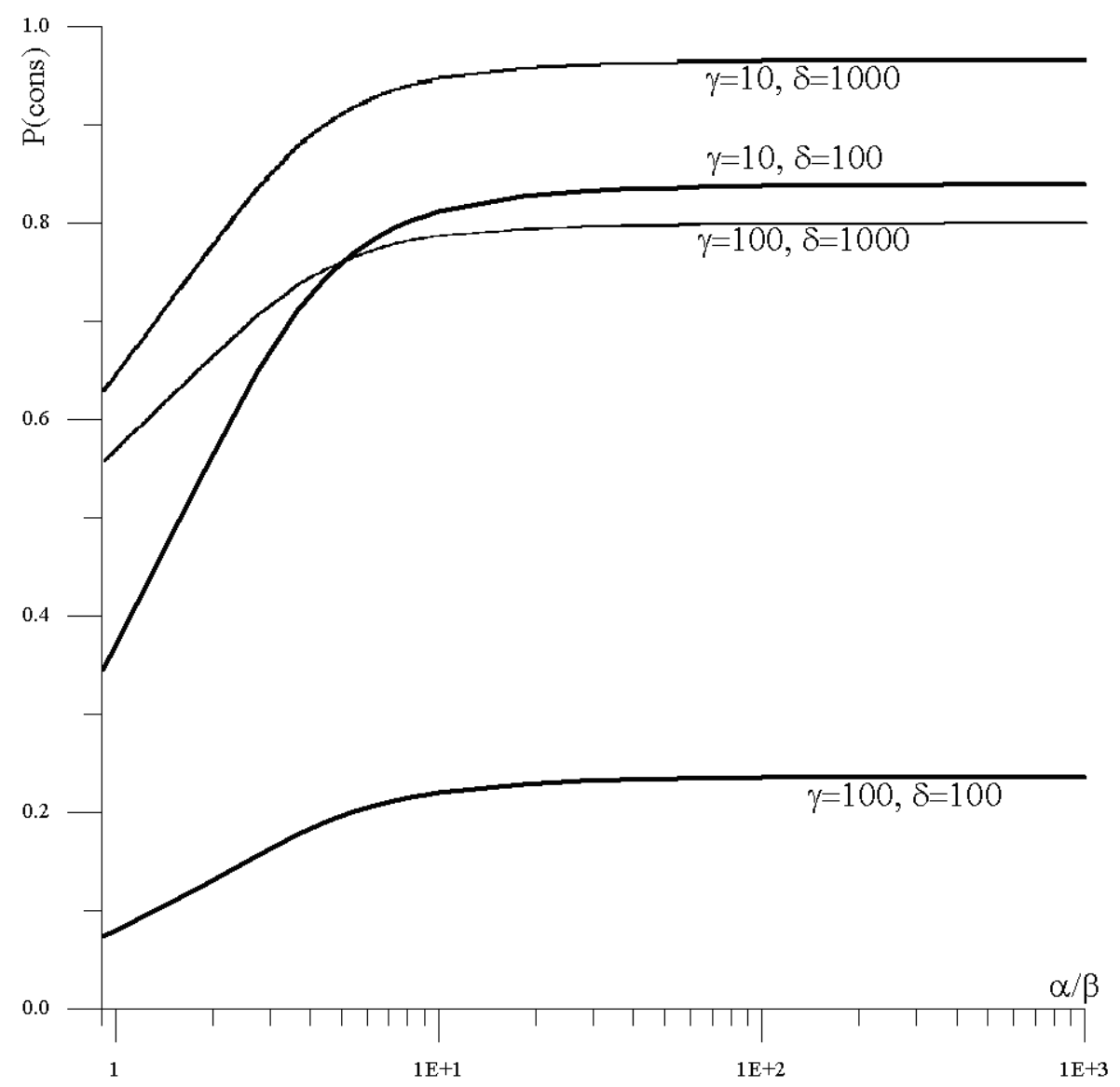

Fig. 7. Probability of having a fully consistent group for $n=k=5$

Considering the behavior of the consistency probability as a function of the replica group size, Fig. 8 shows that for traffic situations corresponding to small values of the ratio $\alpha / \beta$ (e.g., in highway systems) the probability of achieving a full replica group is reduced significantly when the desired group size grows. For the desired group size of $k=7$ in a network with $n=7$ nodes the ratio between $\alpha$ and $\beta$ must be larger than 3 to get a probability of more than $80 \%$ of achieving a full replica group. The above estimates of input values $(\alpha / \beta \leq 3)$, the probability to fill up groups larger than $k=5$ nodes decreases.

With respect to the probability of all replicas being consistent the probability is high even with the ratio between $\gamma$ and $\delta$ being as low as 10, the probability of having consistent replicas is about $80 \%$. Citing the limits given above in Table 1, when considering the extreme high rates and the extreme low rates, the ratio between both $\gamma$ and $\delta$ is equal to 10 . Overall the probability of achieving consistent replica group members is high when the network is fast and not congested. Furthermore the data change rate $\gamma$ must be considerably lower than the update transmission rate. 


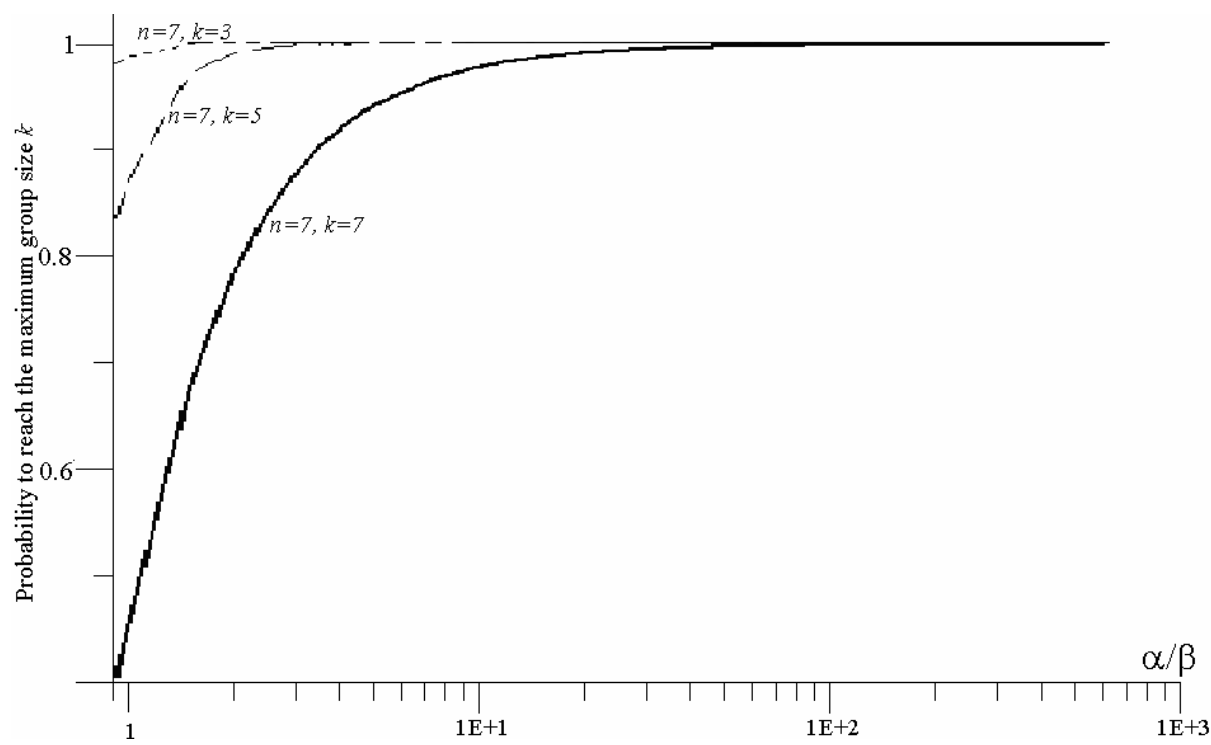

Fig. 8. Probability of reaching different maximum group size $k$ with $n=7$

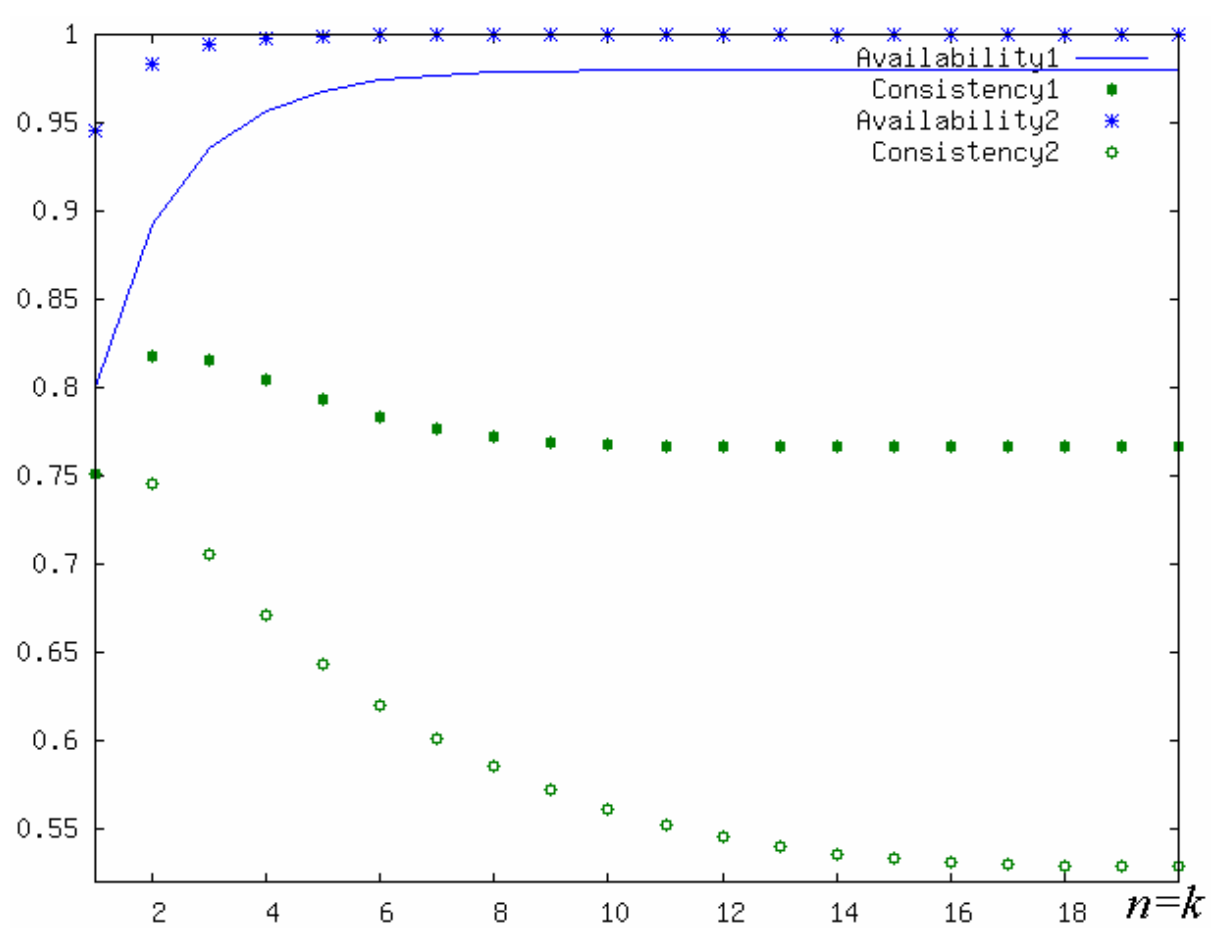

Fig. 9. Service availability vs. group consistency for different group sizes 


\subsection{Service Availability Analyses}

Considering the analytical expression given by Eq. (3) and assuming that each server node in a group has an availability $\mathrm{A}_{\mathrm{s}}=0.6$. Figure 10 shows the service availability for two different settings of $\alpha, \beta, \gamma, \delta$. In the first setting, the parameters are set as follows: $\alpha=5, \beta=1, \delta=100, \gamma=10$. In the second setting the parameters are set as follows: $\alpha=10, \beta=1, \delta=100, \gamma=20$. From the figure it shows that there is a tradeoff between service availability and service correctness (here measured through data consistency). In Figure 9, $n=k$ and it goes from 1 to 20 along the x-axis. It appears that, for the considered numerical values, the best group size providing the best tradeoff between availability and correctness is 3 to 4 servers.

\section{Conclusion and Outlook}

In this paper, we have presented an analytical modeling study based on stochastic Petri nets and Markov chains that allowed us to analyze the behavior of a replication middleware service in ad-hoc based dynamic environments, considering quality of service and dependability related metrics. The main metrics concern the probability of having consistent replicas, the probability of reaching the maximum group size, and service availability. In particular, we carried out several sensitivity analysis studies to see how data consistency is affected in a broad range of scenarios. We can see that the probability to achieve a full group decreases significantly for group sizes with four or more replicas per group. Even though the group will not grow to its full size, the probability of having consistent replicated data is above $80 \%$ in case that the update rate is more than ten times faster than the data change rate. Recall that the data change rate represents the time between two data change events and the update rate represents the time it takes to send an update message to the replica node. With a replica group size of three the probability of reaching a full replica group is almost $50 \%$ higher when $\alpha$ is 100 times bigger than $\beta$.

Moreover we have shown that as service availability increases with a higher number of replicas, the service correctness starts to decrease if the group size goes beyond 4 servers.

The model presented in this paper assumes perfect replica selection and no signaling overhead when exchanging servers in the replica groups. This assumption is limiting the precision of the model but the trends shown in the results section in this paper are valid but optimistic with respect to the level of inconsistency. Another limitation of the model is that it does not consider events like network congestion. In a sense network failures and congestion events can be considered as an increase in the $\beta$ parameter. The selection parameters and algorithms that should be used to select appropriate replica servers are developed in order to ensure maximum stability in the selected replica peers. The exact amount of signaling overhead involved in keeping the participating server nodes and the user nodes up to date on the current group member list is left for future studies. 


\section{References}

[1] SAF, Service Availability Forum, http: //www. saforum. org

[2] Artimy, M.M., Robertson, W., Phillips, W.J.: Connectivity in inter-vehicle ad hoc networks, Faculty of engineering, Dalhouse University (2004)

[3] HIDENETs, Highly Dependable ip-based networks and services, in Project Deliverable D1.2, IST-FP6-STREP-26979 (June 2003)

[4] Avizienis, A., Laprie, J.-C., Randell, B., Landwehr, C.: Basic concepts and taxonomy of dependable and secure computing. IEEE Transactions on Dependable and Secure Computing 1, 11-33 (2004)

[5] Bozinovski, M., Schwefel, H.-P., Prasad, R.: Algorithm for Controlling Transaction Consistency in SIP Session Control Systems. IEE Electronics Letters 40, 209-211 (2004)

[6] Camp, T., Boleng, J., Davies, V.: A survey of mobility models for ad hoc network research. In: Wireless Communications and Mobile Computing (WCMC) (2002)

[7] Chen, I.-R., Baoshan, G., George, S., Sheng-Tzong, C.: On failure recoverability of client-server applications in mobile wireless environments. IEEE Transactions in Reliability 54, 115-122 (2005)

[8] Matthiesen, E.V., Renier, T., Schwefel, H.-P.: A new selection metric for backup group creation in inter-vehicular networks. In: 16th IST Mobile and communications summit (2007)

[9] Chen, Z.D., Kung, H., Vlah, D.: Ad hoc relay wireless networks over moving vehicles on highways. In: MobiHoc 2001: Proceedings of the 2nd ACM international symposium on Mobile ad-hoc networking \& computing, pp. 247-250. ACM Press, New York (2001)

[10] Hansen, M.B., Olsen, R.L., Schwefel, H.-P.: Probabilistic models for access strategies to dynamic information elements. Performance Evaluation (to appear)

[11] Helal, A., Heddaya, A., Bhargava, B.: Replication Techniques in Distributed Systems. Kluwer Academic Publishers, Dordrecht (1996)

[12] Olesen, R.L., Hansen, M.B., Schwefel, H.-P.: Quantitative analysis of access strategies to remote information in network services. In: Global Telecommunications Conference, GLOBECOM - IEEE (2006)

[13] Killijian, M.-O., Powell, D., Banâtre, M., Couderc, P., Roudier, Y.: Collaborative Backup for Dependable Mobile Applications. In: Proc. of 2nd Int. Workshop on Middleware for Pervasive and Ad-Hoc Computing (Middleware 2004), October 2004, pp. 146-149 (2004)

[14] Cox, D.R., Miller, H.D.: The Theory of Stochastic Processes. Chapman and Hall, Boca Raton (1965)

[15] Marsan, M.A., Balbo, G., Conte, G., Donatelli, S., Franceschinis, G.: Modeling with Generalized Stochastic Petri Nets. John Wiley \& Sons, Chichester (1995)

[16] Bolch, G., Greiner, S., de Meer, H., Trivedi, K.S.: Queuing Networks and Markov Chains. Wiley, Chichester (2006) 